\title{
Weighted inequalities for rough square functions through extrapolation
}

by

Javier DuoAndikoetxea (Bilbao) and Edurne SeiJo (Donostia)

\begin{abstract}
Weighted inequalities for some square functions are studied. $L^{2}$ results are proved first using the particular structure of the operator and then extrapolation of weights is applied to extend the results to other $L^{p}$ spaces. In particular, previous results for square functions with rough kernel are obtained in a simpler way and extended to a larger class of weights.
\end{abstract}

1. Introduction. Littlewood-Paley and Marcinkiewicz type operators are given by square functions. For a historical overview of this topic and its several aspects the reader can consult the paper by E. Stein [15]. A typical case is the following: given a function $N$ in $\mathbb{R}^{n}$ satisfying appropriate conditions, define $N_{t}(x)=t^{-n} N\left(t^{-1} x\right)$ for $t>0$ and construct the square function

$$
g(f)(x)=\left(\int_{0}^{\infty}\left|N_{t} * f(x)\right|^{2} \frac{d t}{t}\right)^{1 / 2} .
$$

Although they were originally concerned with dyadic decompositions of Fourier series and integrals, we use the term Littlewood-Paley square functions (in continuous form) for those $g$ obtained by taking $N \in \mathcal{S}$ (the Schwartz class of functions) with $\widehat{N}(0)=0$. In this case,

$$
\|g(f)\|_{p} \leq C\|f\|_{p}, \quad 1<p<\infty .
$$

When $n=1$ and $N(x)=\chi_{[-1,0]}(x)-\chi_{[0,1]}(x), g(f)$ becomes the Marcinkiewicz operator

$$
g(f)(x)=\left(\int_{0}^{\infty}|F(x+t)-2 F(x)+F(x-t)|^{2} \frac{d t}{t^{3}}\right)^{1 / 2}
$$

2000 Mathematics Subject Classification: 42B25, 42B20.

Key words and phrases: square functions, weighted inequalities, extrapolation.

Both authors supported in part by grant EB051/99 of the University of the Basque Country (UPV-EHU), Spain. 
where $F$ is a primitive of $f$. That this operator also satisfies (1) was first proved by Zygmund in 1944. As a generalization of the Marcinkiewicz operator to higher dimensions, Stein [14] proposed the choice

$$
N(x)=|x|^{1-n} \Omega\left(x^{\prime}\right) \chi_{\{|x| \leq 1\}}(x)
$$

where $x^{\prime}=|x|^{-1} x$ and $\Omega$ defined on the unit sphere is a Lipschitz function with zero integral (in fact, the conditions on $\Omega$ were related to the conditions required on the kernel of a smooth singular integral of Calderón-Zygmund type). In that paper he proved that (1) holds for the square function if $1<p \leq 2$, and the result was extended by A. Benedek, A. Calderón, and R. Panzone [1] to $1<p<\infty$ assuming that $\Omega$ is $\mathcal{C}^{1}$. Later on the case of rough kernels $(\Omega$ satisfies only size and cancellation conditions but no regularity is assumed) became of interest for singular integral operators and the analogous operator was also defined for square functions.

D. Kurtz [11] proved that for the Littlewood-Paley case, (1) holds also in $L^{p}(w)$, the $L^{p}$ space with respect to the measure $w(x) d x$, when $1<p<\infty$ and $w \in A_{p}$ (the Muckenhoupt class of weights for which the HardyLittlewood maximal operator is bounded in $L^{p}(w)$ ). We will use this result in our approach. In [19] the square operators defined by Stein were studied and among other interesting results weighted inequalities were proved: again boundedness holds on $L^{p}(w)$ for $w \in A_{p}$ when $1<p<\infty$. In a recent paper, Y. Ding, D. Fan, and Y. Pan [3] have studied weighted inequalities for square functions with rough kernel. If $\Omega \in L^{q}\left(S^{n-1}\right)$ they proved the boundedness for $w \in A_{p / q^{\prime}}$ for $p>q^{\prime}$ and dual-type results for smaller values of $p$ as had previously been obtained for similar singular integral operators. For the definition and properties of $A_{p}$ weights see [9], for instance.

The aim of this paper is to study weighted inequalities for a large class of square functions. Due to the special feature of the operator, it is well adapted to deducing weighted inequalities in the case $p=2$. Our first main result is that assuming some decay properties on the Fourier transform of $N_{t}$ (which we do not take in general as dilations of a fixed $N$ ), the square function is bounded on $L^{2}\left(w^{s}\right)$ if $w \in A_{2}$ is a uniform weight for the operators given by convolution with $N_{t}$ and $0 \leq s<1$. Then we introduce a powerful tool: extrapolation. This tool has been missed in the preceding approaches or used only with $A_{p}$ classes ([13]), which limits its strength.

Following the spirit of the first extrapolation theorem by J. L. Rubio de Francia [12], $L^{p}$-weighted inequalities for a large class of weights are deduced from $L^{2}$-weighted inequalities. In particular, the case of $\Omega \in L^{\infty}$ (which includes $\Omega$ smooth) is trivially deduced from the extrapolation theorem of Rubio de Francia.

The paper is organized as follows. In Section 2 we prove the $L^{2}$ result; in Section 3 we state some $L^{p}$ results for weights related to a maximal 
function and in Section 4 we present some applications which cover and extend previously known results. In Section 5 we deal with a more singular case related to spherical means which has the drawback of the behaviour of the spherical maximal function; nevertheless we show that even in this case our method is useful. Finally in Section 6 we show some further extensions.

We denote by $\|f\|_{p, w}$ the norm of $f$ in $L^{p}(w)$ and by $A^{r} B^{s}$ the set $\left\{u^{r} v^{s}: u \in A, v \in B\right\}$. $M$ will always be used to denote the HardyLittlewood maximal operator.

\section{2. $L^{2}$ theorems}

Theorem 1. Assume that for each $t>0$ and for some $\alpha, \beta>0$,

$$
\left|\widehat{N}_{t}(\xi)\right| \leq C \min \left(|t \xi|^{-\alpha},|t \xi|^{\beta}\right)
$$

and that $w \in A_{2}$ satisfies

$$
\sup _{t>0} \int_{\mathbb{R}^{n}}\left|N_{t} * f(x)\right|^{2} w(x) d x \leq C \int_{\mathbb{R}^{n}}|f(x)|^{2} w(x) d x .
$$

Then, if $0 \leq s<1$,

$$
\int_{\mathbb{R}^{n}}|g(f)(x)|^{2} w^{s}(x) d x \leq C \int_{\mathbb{R}^{n}}|f(x)|^{2} w^{s}(x) d x .
$$

Proof. Let $\psi \in \mathcal{S}\left(\mathbb{R}^{n}\right)$ be a radial function with $\operatorname{supp} \widehat{\psi} \subset\{\xi: 1 / 2<$ $|\xi| \leq 2\}$ and such that $\sum_{k=-\infty}^{\infty} \widehat{\psi}\left(2^{k} \xi\right)=1$ for all $\xi \neq 0$. For each integer $k$ and for $t>0$ define $\left(\psi_{k}\right)_{t}$ as the function satisfying $\left(\widehat{\left.\psi_{k}\right)_{t}}(\xi)=\widehat{\psi}\left(2^{k} t \xi\right)\right.$. Then for each $t>0$ we can write

$$
N_{t} * f(x)=\sum_{k=-\infty}^{\infty} N_{t} *\left(\psi_{k}\right)_{t} * f(x) .
$$

Let $g_{k}(f)$ be the square function associated with $N_{t} *\left(\psi_{k}\right)_{t}$, that is,

$$
g_{k}(f)(x)=\left(\int_{0}^{\infty}\left|N_{t} *\left(\psi_{k}\right)_{t} * f(x)\right|^{2} \frac{d t}{t}\right)^{1 / 2} .
$$

Then using Minkowski's inequality we have

$$
\|g(f)\|_{p, w} \leq \sum_{k=-\infty}^{\infty}\left\|g_{k}(f)\right\|_{p, w} .
$$

We first compute the $L^{2}$ norm of $g_{k}$; using the Fubini and Plancherel theorems we get

$$
\left\|g_{k}(f)\right\|_{2}^{2}=\int_{0}^{\infty} \int_{\mathbb{R}^{n}}\left|\widehat{N}_{t}(\xi)\right|^{2}\left|\widehat{\psi}\left(2^{k} t \xi\right)\right|^{2}|\widehat{f}(\xi)|^{2} d \xi \frac{d t}{t} .
$$


Using now the support of $\psi$ and condition (2) on $N_{t}$ we get

$$
\begin{aligned}
\left\|g_{k}(f)\right\|_{2}^{2} & \leq C \int_{\mathbb{R}^{n}}^{2^{-k+1}|\xi|^{-1}} \int_{2^{-1}|\xi|^{-1}}\left(\min \left(|t \xi|^{-\alpha},|t \xi|^{\beta}\right)\right)^{2} \frac{d t}{t}|\widehat{f}(\xi)|^{2} d \xi \\
& \leq C 2^{-2|k| \sigma} \int_{\mathbb{R}^{n}}|f|^{2}
\end{aligned}
$$

where $\sigma=\min (\alpha, \beta)$. Therefore, for all integers $k$,

$$
\left\|g_{k}(f)\right\|_{2} \leq C 2^{-|k| \sigma}\|f\|_{2} \quad \text { with } \sigma>0 .
$$

We now find a weighted estimate for $g_{k}(f)$ in $L^{2}(w)$. Fix $w \in A_{2}$. Then

$$
\begin{aligned}
\left\|g_{k}(f)\right\|_{2, w}^{2} & =\int_{0}^{\infty} \int_{\mathbb{R}^{n}}\left|N_{t} *\left(\psi_{k}\right)_{t} * f(x)\right|^{2} w(x) d x \frac{d t}{t} \\
& \leq C \int_{\mathbb{R}^{n}} \int_{0}^{\infty}\left|\left(\psi_{k}\right)_{t} * f(x)\right|^{2} \frac{d t}{t} w(x) d x \\
& \leq C \int_{\mathbb{R}^{n}}|f(x)|^{2} w(x) d x
\end{aligned}
$$

where the first inequality is due to (3) and for the second one we use the weighted estimate for the Littlewood-Paley square function with $w \in A_{2}$, mentioned in the introduction and due to Kurtz.

Apply interpolation with change of measure (see [18]) to this uniform weighted estimate and to (5) to get, for $0 \leq s<1$,

$$
\left\|g_{k}(f)\right\|_{2, w^{s}} \leq C 2^{-|k| \sigma(1-s)}\|f\|_{2, w^{s}}
$$

Summing over $k$ we deduce the theorem.

Remarks. 1. From the Plancherel theorem it is immediate that

$$
\sup _{\xi} \int_{0}^{\infty}\left|\widehat{N}_{t}(\xi)\right|^{2} \frac{d t}{t}<\infty
$$

is necessary and sufficient for the $L^{2}$ boundedness of $g$. Nevertheless this condition is too general to deduce other boundedness properties from it.

2. Condition (2) is simple to state and works in all our examples but the proof of the theorem shows that it can be replaced by a more general one:

$$
\sup _{u \in S^{n-1}} \frac{1}{R} \int_{R}^{2 R}\left|\widehat{N}_{t}(u)\right|^{2} d t \leq C \min \left(R, R^{-1}\right)^{\sigma} \quad \text { for some } \sigma>0 .
$$

3. If $N_{t}(x)=t^{-n} N\left(t^{-1} x\right)$, (2) reduces to $|\widehat{N}(\xi)| \leq \min \left(|\xi|^{-\alpha},|\xi|^{\beta}\right)$. 
3. $L^{p}$ results through extrapolation. Assume that (3) is satisfied by all $A_{2}$ weights; then the same is true for the conclusion of Theorem 1 in view of the property $A_{q}=\bigcup_{0<s<1}\left\{w^{s}: w \in A_{q}\right\}$ of this class of weights. Then using the extrapolation theorem of Rubio de Francia $([12,9])$ we deduce that $g$ is bounded on $L^{p}(w)$ for $w \in A_{p}$. In a similar way, if (3) is satisfied by all $A_{r}$ weights $(r<2)$ then by extrapolation $g$ is bounded on $L^{p}(w)$ for $w \in A_{p / r}$ when $p>r$. But the scope of the extrapolation theorem is larger than $A_{p}$ weights, and in this section we give a result which applies in greater generality.

Given an operator $T$ we define, for $1<p<\infty$,

$$
W_{p}(T)=\left\{w: \int_{\mathbb{R}^{n}}|T f|^{p} w \leq C \int_{\mathbb{R}^{n}}|f|^{p} w\right\}
$$

and for $p=1$,

$$
W_{1}(T)=\{w: T w(x) \leq C w(x) \text { a.e. }\} .
$$

We also define, for $1 \leq p<\infty$,

$$
A W_{p}(T)=\left\{w \in W_{p}(T): w^{s} \in W_{p}(T) \text { for some } s>1\right\} .
$$

When applied to the Hardy-Littlewood maximal function, this class is the same as $A_{p}$ but in general $A W_{p}(T)$ can be strictly contained in $W_{p}(T)$.

In order to get weights for which (3) holds, we can proceed as follows. Assume $\mathcal{M}$ is a positive sublinear operator and $C$ a constant such that

$$
\sup _{t>0}\left|N_{t} * f(x)\right| \leq C \mathcal{M}(|f|)(x) \quad \text { a.e. }
$$

It is clear that weights in $W_{2}(\mathcal{M})$ satisfy $(3)$. Thus the conclusion of Theorem 1 is valid for $\left\{w^{s}: w \in W_{2}(\mathcal{M}+M), 0 \leq s<1\right\}$. This observation is enough for the applications of the following section.

A possibly larger class of weights satisfying (3) can be obtained. We first recall a result for positive linear operators. Let $S$ be a positive linear operator and $S^{*}$ its adjoint, and let $w_{0}, w_{1}$ be such that

$$
S w_{1} \leq B_{1} w_{1} \quad \text { a.e., } \quad S^{*} w_{0} \leq B_{0} w_{0} \quad \text { a.e. }
$$

Then $S$ is bounded on $L^{p}\left(w_{0} w_{1}^{1-p}\right)$ with a bound depending only on $B_{0}$ and $B_{1}$. (This follows by interpolation since (7) implies that $f \mapsto w_{1}^{-1} S\left(w_{1} f\right)$ is bounded on $L^{1}\left(w_{0} w_{1}\right)$ and on $L^{\infty}\left(w_{0} w_{1}\right)$.) The converse is also true but we only need this easy part of the factorization in what follows.

Let $S_{t}$ be the linear positive operator defined by convolution with $\left|N_{t}\right|$ and $S_{t}^{*}$ its adjoint (given by convolution with $\left|N_{t}(-x)\right|$ ). If $\sup _{t}\left|S_{t} f\right| \leq$ $\mathcal{M} f$ and $\sup _{t}\left|S_{t}^{*} f\right| \leq \mathcal{M}^{*} f$ then according to the result for positive linear operators, (3) holds for all weights in the class $W_{1}\left(\mathcal{M}^{*}\right) W_{1}(\mathcal{M})^{-1}$. Thus the conclusion of Theorem 1 holds when $w$ is in $A W_{1}\left(\mathcal{M}^{*}+M\right) A W_{1}(\mathcal{M}+M)^{-1}$. This result will be used in the example studied in Section 5 . 
In many cases, weights in these classes are adequate to use extrapolation theorems; the following is a useful version for us.

Theorem 2. Assume that $\mathcal{M}$ and $\mathcal{M}^{*}$ are two positive sublinear operators such that $1 \in A W_{1}\left(\mathcal{M}^{*}\right) \cap A W_{1}(\mathcal{M})$ and for all $1<p<\infty$,

$$
\begin{aligned}
& A W_{1}\left(\mathcal{M}^{*}\right) A W_{1}(\mathcal{M})^{1-p}=A W_{p}(\mathcal{M}), \\
& A W_{1}(\mathcal{M}) A W_{1}\left(\mathcal{M}^{*}\right)^{1-p}=A W_{p}\left(\mathcal{M}^{*}\right) .
\end{aligned}
$$

If $T$ is an operator which is bounded on $L^{2}(w)$ for all $w \in A W_{2}(\mathcal{M})$, then $T$ is bounded on $L^{p}(w)$ for all $w$ in $A W_{p}(\mathcal{M})$.

The proof of the theorem is essentially the same as the proof of the extrapolation theorem given by Rubio de Francia [12] or by Jawerth [10].

Corollary 3. If $N_{t}$ satisfies (2) and if there exist operators $\mathcal{M}$ and $\mathcal{M}^{*}$ such that (6), (8), and (9) hold, then the square function $g$ is bounded on $L^{p}(w)$ for all weights $w$ in $A W_{p}(\mathcal{M}+M)$.

For the rough operators in the next section the conditions of the corollary appear in [20]. In a more abstract setting the following result is given in [6]: let $\left\{\mu_{j}\right\}_{j \in \mathbb{Z}}$ be a sequence of positive Borel measures and $\mathcal{M}$ the maximal operator $\mathcal{M} f(x)=\sup _{j \in \mathbb{Z}}\left|\mu_{j} * f(x)\right|$; if for all $j \in \mathbb{Z}$,

$$
\begin{aligned}
\left|\widehat{\mu}_{j}(\xi)\right| & \leq C\left|2^{j} \xi\right|^{-a} \quad \text { for some } a>0, \\
\left|\widehat{\mu}_{j}(\xi)-\widehat{\mu}_{j}(0)\right| & \leq C\left|2^{j} \xi\right|,
\end{aligned}
$$

and $\left\{\widehat{\mu}_{j}(0)\right\}_{j \in \mathbb{Z}}$ are uniformly bounded, then the factorization properties hold for $\mathcal{M}$ and $\mathcal{M}^{*}$ (defined using the adjoint operators).

4. Some applications. Let $\Omega$ be a function defined on the unit sphere $S^{n-1}$ which is in $L^{q}\left(S^{n-1}\right)$ for some $q>1$ and has mean value zero; for each $t>0$, let $b_{t}$ be a bounded radial function with bound uniform in $t$ and $\beta<n$. Define the square function

$$
g(f)(x)=\left(\int_{0}^{\infty}\left|\frac{1}{t^{n-\beta}} \int_{|y| \leq t} b_{t}(|y|) \frac{\Omega\left(y^{\prime}\right)}{|y|^{\beta}} f(x-y) d y\right|^{2} \frac{d t}{t}\right)^{1 / 2},
$$

and consider the maximal operator

$$
M_{\Omega} f(x)=\sup _{r>0} \frac{1}{r^{n}} \int_{|y| \leq r}\left|\Omega\left(y^{\prime}\right)\right| \cdot|f(x-y)| d y .
$$

Let $\mathcal{M}=M_{\Omega}+M$ and $\mathcal{M}^{*}=M_{\widetilde{\Omega}}+M$ where $\widetilde{\Omega}(u)=\Omega(-u)$ and $M$ is the Hardy-Littlewood maximal function. Then (8) and (9) hold. 
THEOREM 4. With the above notation and assumptions on $\Omega$ and $b_{t}$, the weighted norm inequality

$$
\|g(f)\|_{p, w} \leq C\|f\|_{p, w}
$$

holds for $1<p<\infty$ and all weights in $A W_{p}\left(M_{\Omega}+M\right)$.

Proof. Define

$$
N_{t}(x)=\frac{1}{t^{n-\beta}} b_{t}(|x|) \frac{\Omega\left(x^{\prime}\right)}{|x|^{\beta}} \chi_{\{|x| \leq t\}} .
$$

To prove the theorem we only need to check (2) and the majorization by $\mathcal{M}$. The latter comes from

$$
\begin{aligned}
|| N_{t}|* f(x)| & \leq \frac{1}{t^{n-\beta}} \int_{|y| \leq t}\left|b_{t}(|y|)\right| \frac{\left|\Omega\left(y^{\prime}\right)\right|}{|y|^{\beta}}|f(x-y)| d y \\
& \leq\left\|b_{t}\right\|_{\infty} \sum_{k=1}^{\infty} \frac{2^{(k+1) \beta}}{t^{n}} \int_{2^{-k-1} t<|y| \leq 2^{-k} t}\left|\Omega\left(y^{\prime}\right)\right| \cdot|f(x-y)| d y \\
& \leq C(\beta)\left\|b_{t}\right\|_{\infty} M_{\Omega} f(x)
\end{aligned}
$$

with $C(\beta)$ finite if $\beta<n$.

Concerning the bounds of the Fourier transform we have

$$
\begin{aligned}
\left|\widehat{N}_{t}(\xi)\right| & =\left|\int_{|x| \leq t} b_{t}(|x|) \frac{\Omega\left(x^{\prime}\right)}{t^{n-\beta}|x|^{\beta}}\left(e^{-2 \pi i x \cdot \xi}-1\right) d x\right| \\
& \leq 2 \pi|\xi| \cdot\left\|b_{t}\right\|_{\infty} \int_{|x| \leq t} \frac{\left|\Omega\left(x^{\prime}\right)\right|}{t^{n-\beta}|x|^{\beta-1}} d x \leq C t|\xi|
\end{aligned}
$$

using the fact that $\Omega$ has integral zero. On the other hand,

$$
\begin{aligned}
\left|\widehat{N}_{t}(\xi)\right| & =\left|\frac{1}{t^{n-\beta}} \int_{0}^{t} b_{t}(r) r^{n-1-\beta}\left(\int_{S^{n-1}} \Omega(u) e^{-2 \pi i r u \cdot \xi} d \sigma(u)\right) d r\right| \\
& =\left|\int_{0}^{1} b_{t}(t r) r^{n-1-\beta}\left(\int_{S^{n-1}} \Omega(u) e^{-2 \pi i r u \cdot t \xi} d \sigma(u)\right) d r\right| .
\end{aligned}
$$

Define $I_{r}(\xi)=\int_{S^{n-1}} \Omega(u) e^{-2 \pi i r u \cdot \xi} d \sigma(u)$. Then

$$
\begin{aligned}
\left|\widehat{N}_{t}(\xi)\right|^{2} & \leq C_{\beta}\left\|b_{t}\right\|_{\infty} \int_{0}^{1} r^{n-1-\beta}\left|I_{r}(t \xi)\right|^{2} d r \\
& =C_{\beta}\left\|b_{t}\right\|_{\infty} \int_{0}^{1} r^{n-1-\beta} \iint_{S^{n-1} \times S^{n-1}} \Omega(u) \Omega(v) e^{-2 \pi i r(u-v) \cdot t \xi} d \sigma(u) d \sigma(v) d r .
\end{aligned}
$$


Now for $n-1<\beta<n$, we have

$$
\left|\int_{0}^{1} r^{n-1-\beta} e^{-i r u \cdot \xi} d r\right| \leq C \min \left(1,|u \cdot \xi|^{\beta-n}\right) \leq C\left(\left|u \cdot \xi^{\prime}\right| \cdot|\xi|\right)^{(\beta-n) \varepsilon}
$$

for any $0 \leq \varepsilon \leq 1$, and for $\beta \leq n-1$,

$$
\left|\int_{0}^{1} r^{n-1-\beta} e^{-i r u \cdot \xi} d r\right| \leq C \min \left(1,|u \cdot \xi|^{-1}\right) \leq C\left(\left|u \cdot \xi^{\prime}\right| \cdot|\xi|\right)^{-\varepsilon} .
$$

The estimate with an absolute constant is immediate and to get the bound $C|u \cdot \xi|^{\beta-n}$ in (11) when $|u \cdot \xi|$ is large, split the integral into two parts, from 0 to $A$ and from $A$ to 1 . For the first part the bound $C A^{n-\beta}$ is immediate and in the second one integration by parts gives $C A^{n-\beta-1}|u \cdot \xi|^{-1}$; choose then $A=|u \cdot \xi|^{-1}$. To prove the bound $C|u \cdot \xi|^{-1}$ when $\beta<n-1$ it is enough to integrate by parts due to the integrability of $r^{n-2-\beta}$.

When $n-1<\beta<n$, using (11) we have

$$
\begin{aligned}
\left|\widehat{N}_{t}(\xi)\right|^{2} \leq & C_{\beta}\left\|b_{t}\right\|_{\infty}|t \xi|^{(\beta-n) \varepsilon} \\
& \times \int_{S^{n-1} \times S^{n-1}}|\Omega(u) \Omega(v)| \cdot\left|(u-v) \cdot \xi^{\prime}\right|^{(\beta-n) \varepsilon} d \sigma(u) d \sigma(v) .
\end{aligned}
$$

Apply Hölder's inequality and choose $\varepsilon$ so that $(n-\beta) \varepsilon q^{\prime}<1$ to get the desired bound. When $\beta \leq n-1$ use (12) and choose $\varepsilon<1 / q^{\prime}$.

This example covers several situations. If $b_{t} \equiv 1$ we get Stein's square function as mentioned in Section 1 but with $\Omega \in L^{q}\left(S^{n-1}\right)$ and no regularity assumption. But we can also introduce a fixed bounded function $b$ in the kernel $N$ and define $N_{t}$ by dilation; then $b_{t}(|x|)=b\left(t^{-1}|x|\right)$. The case studied in [3] corresponds to $b_{t}$ independent of $t$. In all cases the weights are given by the corresponding weights for $M_{\Omega}$. All the results described in [3] are immediately included (see [20] and [5]): $w \in A_{p / q^{\prime}}$ for $p \geq q^{\prime}$ and $w^{1-p}$ for $w \in A_{p^{\prime} / q^{\prime}}$ and $1<p \leq q$ satisfy the condition of Theorem 4 because they are weights for $M_{\Omega}$. For power weights of the form $w(x)=|x|^{\alpha}$ the operator is bounded if

$$
\max \left(-n,-1-(n-1) p / q^{\prime}\right)<\alpha<\min \left(n(p-1), p-1+(n-1) p / q^{\prime}\right)
$$

(see [5]), and this result is better than the one given by the $A_{p}$ type conditions in $[3]$.

Without any substantial modification of the proof of the estimate for the Fourier transform of $N_{t}$, a more general condition on $b_{t}$ is possible when it is independent of $t$, namely,

$$
\sup _{t} \frac{1}{t^{n-\beta}} \int_{0}^{t}|b(r)|^{2} r^{n-1-\beta} d r<\infty
$$


But in this case, the maximal function which controls $N_{t}$ is not $M_{\Omega}$ and has to be modified to the following one:

$$
M_{\Omega, b}(f)(x)=\sup _{r>0} \frac{1}{r^{n}} \int_{|y| \leq r}\left|b(|y|) \Omega\left(y^{\prime}\right) f(x-y)\right| d y .
$$

That this maximal function also satisfies the factorization conditions (8) and (9) is deduced from the decay properties of the Fourier transform of its dyadic decomposition by applying the result of [6] mentioned after Corollary 3.

Another application of this method gives an improvement of Theorem 2 in Sato's paper [13]. The operator is given through dilations of a fixed $N$ and two technical conditions are enough to insure the $L^{2}$ result (instead of (2) we would use Remark 2 following Theorem 1); then he introduces the following hypotheses: $|N(x)| \leq b(|x|) \Omega\left(x^{\prime}\right)$ where $b$ is non-increasing in $(0, \infty)$ and $B(x)=b(|x|)$ is integrable, and $\Omega \in L^{q}\left(S^{n-1}\right)$ for $2 \leq q \leq \infty$. The conclusion is that the square function is bounded on $L^{p}(w)$ for $w \in A_{p / q^{\prime}}$ when $p>q^{\prime}$. Due to the pointwise bound on $N$ it is easy to check that convolution with $N_{t}$ is controlled by the maximal operator $M_{\Omega}$ defined in (10). Using Theorem 1 and extrapolation we deduce that the square function is bounded on $L^{p}(w)$ for all $w \in A W_{p}\left(M_{\Omega}+M\right)$; thus the assumption $q>2$ in Sato's paper is unnecessary, but even in that case a larger class of weights is obtained, as in the previous example.

5. A more singular example. Let $N(x)=\Omega\left(x^{\prime}\right) d \sigma(x)$ where $\Omega$ is a $\mathcal{C}^{\infty}$ function defined on the unit sphere with mean value zero and $d \sigma$ is Lebesgue measure on $S^{n-1}$. Define $N_{t}$ by dilation in such a way that $N_{t}(x)=\Omega\left(x^{\prime}\right) d \sigma_{t}(x)$ where $d \sigma_{t}$ is the normalized Lebesgue measure on the sphere of radius $t$. Then $N_{t}$ is a singular finite Borel measure and not a function. Let $g$ be the square function defined with such a family $N_{t}$.

The maximal operator $\mathcal{M}$ which controls convolution with $\left|N_{t}\right|$ will be the spherical maximal function, that is, the maximal function defined as the supremum over all means on spheres centered at each point. This maximal operator is only bounded when $p>n /(n-1)$; its weighted inequalities are only partially known and we will use results proved in [8], in particular $A W_{p}(\mathcal{M}) \subset A_{p}$. We use the same maximal operator to control the adjoints of the convolutions.

THEOREM 5. The square function $g$ satisfies the weighted inequalities

$$
\|g(f)\|_{p, w} \leq C\|f\|_{p, w}
$$

when:

(i) $2 n /(2 n-1)<p \leq 2$ and $w=w_{0} w_{1}^{\left(1-2 n / p^{\prime}\right) /(n-1)}$ with $w_{0} \in A W_{1}(\mathcal{M}) \cap$ $A W_{p /(2-p)}(\mathcal{M})$ and $w_{1} \in A W_{1}(\mathcal{M})$; 
(ii) $2 \leq p<2 n$ and $w=w_{0}^{(2 n / p-1) /(n-1)} w_{1}^{1-p}$ with $w_{0} \in A W_{1}(\mathcal{M})$ and $w_{1} \in A W_{1}(\mathcal{M}) \cap A W_{p /(p-2)}(\mathcal{M})$.

In particular, $g$ is bounded on $L^{p}\left(|x|^{\alpha}\right)$ if $2 n /(2 n-1)<p \leq 2$ and $1-n<\alpha<1-2 n / p^{\prime}$ or if $2<p<2 n$ and $1-2 n / p<\alpha<(n-1)(p-1)$.

If $f$ is the characteristic function of the unit ball and $\Omega$ is 1 on a big portion of the unit sphere, then for large $x$ we have $N_{t} * f(x) \sim|x|^{1-n}$ if $|x|-1 / 2<t<|x|+1 / 2$; the integrability of the square function in $x$ for $w \equiv 1$ requires $p>2 n /(2 n-1)$, which is the restriction of the theorem.

Proof. We first apply Theorem 1. Since the operators $N_{t}$ are dilations of a fixed one, we only need to check the conditions on $N$ (see Remark 3 following Theorem 1). $|\widehat{N}(\xi)| \leq C|\xi|$ is immediate because $\widehat{N}(0)=0$. The decay property $|\widehat{N}(\xi)| \leq C|\xi|^{(1-n) / 2}$ can be seen in [16], p. 348. Using now the spherical maximal function to control the convolution operators we use the discussion of Section 3 to deduce

$$
\|g(f)\|_{2, w} \leq C\|f\|_{2, w}
$$

for all $w \in A W_{1}(\mathcal{M}) A W_{1}(\mathcal{M})^{-1}$.

The main difference with the results of the preceding section is that the factorization used in Theorem 2 is false. Nevertheless the $L^{2}$ result contains enough information to deduce some $L^{p}$ results.

Assume first that $2 n /(2 n-1)<p<2$ and let $f \in L^{p}(w)$ be nonnegative with $w \in A W_{1}(\mathcal{M}) \cap A W_{p /(2-p)}(\mathcal{M})$. There exists $\varepsilon>0$ such that $w^{1+\varepsilon} \in W_{p /(2-p)}(\mathcal{M})$ and since $1 \in W_{q}(\mathcal{M})$ for $n /(n-1)<q<p /(2-p)$, by interpolation with change of measure there exists $s<1$ such that $w \in$ $W_{p s /(2-p)}(\mathcal{M})$. On the other hand, $f^{(2-p) / s} \in L^{p s /(2-p)}(w)$ so that we can use the construction of Rubio de Francia (see [12]) to define

$$
u^{1 / s}=\sum_{k=0}^{\infty} \frac{\mathcal{M}^{k}\left(f^{(2-p) / s}\right)}{(2\|\mathcal{M}\|)^{k}}
$$

where $\|\mathcal{M}\|$ is the norm of $\mathcal{M}$ in $L^{p s /(2-p)}(w)$ and $\mathcal{M}^{k}$ denotes the $k$-fold iteration of $\mathcal{M}$. The function $u$ has the following properties easily deduced from its definition:

$$
|f|^{2-p} \leq u \text { a.e., } \quad\|u\|_{p /(2-p), w} \leq C\|f\|_{p, w}, \quad u^{1 / s} \in W_{1}(\mathcal{M}) .
$$

Then using Hölder's inequality and the fact that $u, w \in A W_{1}(\mathcal{M})$ we have

$$
\begin{aligned}
\int g(f)^{p} w & =\int g(f)^{p} u^{-p / 2} u^{p / 2} w \leq\left(\int g(f)^{2} u^{-1} w\right)^{p / 2}\left(\int u^{p /(2-p)} w\right)^{1-p / 2} \\
& \leq C\left(\int f^{2} u^{-1} w\right)^{p / 2}\left(\int f^{p} w\right)^{1-p / 2} .
\end{aligned}
$$

In the last inequality we also used the second property in (13); using the first one we have $u^{-1} \leq f^{p-2}$ and this proves the boundedness of $g$ on 
$L^{p}(w)$ when $w \in A W_{1}(\mathcal{M}) \cap A W_{p /(2-p)}(\mathcal{M})$. Interpolating with the $L^{2}(w)$ boundedness we deduce the result of (i).

Let now $p>2$ and $w \in A W_{1}(\mathcal{M}) \cap A W_{p /(p-2)}(\mathcal{M})$. Then

$$
\left(\int g(f)^{p} w^{1-p}\right)^{2 / p}=\int\left(g(f) w^{-1}\right)^{2} v w=\int g(f)^{2} v w^{-1}
$$

for some $v \in L^{p /(p-2)}(w)$ with norm 1. Applying again Rubio de Francia's construction as before (to $v^{1 / s} \in L^{p s /(p-2)}(w)$ ) we have

$$
v \leq u \text { a.e., } \quad\|u\|_{p /(p-2), w} \leq C\|v\|_{p /(p-2), w}, \quad u^{1 / s} \in W_{1}(\mathcal{M}) .
$$

Applying the $L^{2}$ result to the weight $u w^{-1}$, Hölder's inequality and the properties of $u$, we deduce the $L^{p}\left(w^{1-p}\right)$ boundedness for $w \in A W_{1}(\mathcal{M}) \cap$ $A W_{p /(p-2)}(\mathcal{M})$. Interpolating again with the $L^{2}(w)$ boundedness for $w \in$ $A W_{1}(\mathcal{M})$ we complete the proof of (ii).

The particular case is deduced from the fact that $|x|^{\alpha} \in W_{1}(\mathcal{M})$ if and only if $1-n \leq \alpha \leq 0$ (see [8]).

It is an open question whether the inclusion $A W_{1}(\mathcal{M}) \subset W_{p}(\mathcal{M})$ holds for the values of $p$ for which the spherical maximal operator is bounded, that is, $p>n /(n-1)$. If the answer were positive, the statement of the theorem would not require the extra assumptions $w_{0} \in A W_{p /(2-p)}(\mathcal{M})$ and $w_{1} \in A W_{p /(p-2)}(\mathcal{M})$.

\section{Further results}

6.1. Product spaces. The general theorems in this work can be extended to product spaces. For simplicity we consider the case of two spaces $\mathbb{R}^{n_{1}} \times \mathbb{R}^{n_{2}}$. The square functions we are interested in are

$$
g(f)\left(x_{1}, x_{2}\right)=\left(\int_{0}^{\infty} \int_{0}^{\infty}\left|N_{t_{1}, t_{2}} * f\left(x_{1}, x_{2}\right)\right|^{2} \frac{d t_{1}}{t_{1}} \frac{d t_{2}}{t_{2}}\right)^{1 / 2} .
$$

In this case the conditions (2) on the Fourier transform of Theorem 1 are replaced by product type conditions

$$
\left|\widehat{N}_{t_{1}, t_{2}}\left(\xi_{1}, \xi_{2}\right)\right| \leq C \min \left(\left|t_{1} \xi_{1}\right|^{-\alpha_{1}},\left|t_{1} \xi_{1}\right|^{\sigma_{1}}\right) \min \left(\left|t_{2} \xi_{2}\right|^{-\alpha_{2}},\left|t_{2} \xi_{2}\right|^{\sigma_{2}}\right)
$$

for $\xi_{1} \in \mathbb{R}^{n_{1}}, \xi_{2} \in \mathbb{R}^{n_{2}}$ and for some positive $\alpha_{1}, \sigma_{1}, \alpha_{2}, \sigma_{2}$. Condition (3) is the same (uniform $L^{2}$ weights for all $N_{t_{1}, t_{2}}$ ) but $w$ must now be in the class $A_{2}^{*}$, which is the class of weights $w$ for which the strong maximal function (the maximal function on cartesian products of balls in $\mathbb{R}^{n_{1}}$ and $\mathbb{R}^{n_{2}}$ ) is bounded on $L^{2}(w)$.

The Marcinkiewicz-type square function with rough kernel in this context will be defined with an $\Omega \in L^{q}\left(S^{n_{1}-1} \times S^{n_{2}-1}\right)$ for some $q>1$ satisfying 
a double cancellation condition

$$
\int_{S^{n_{1}-1}} \Omega\left(y_{1}^{\prime}, y_{2}^{\prime}\right) d \sigma\left(y_{1}^{\prime}\right)=\int_{S^{n_{2}-1}} \Omega\left(y_{1}^{\prime}, y_{2}^{\prime}\right) d \sigma\left(y_{2}^{\prime}\right)=0,
$$

and a bounded family of functions $b_{t_{1}, t_{2}}\left(\left|x_{1}\right|,\left|x_{2}\right|\right)$. Let $\beta_{1}<n_{1}, \beta_{2}<n_{2}$. Then

$$
N_{t_{1}, t_{2}}\left(x_{1}, x_{2}\right)=\frac{b_{t_{1}, t_{2}}\left(\left|x_{1}\right|,\left|x_{2}\right|\right)}{t_{1}^{n_{1}-\beta_{1}} t_{2}^{n_{2}-\beta_{2}}} \cdot \frac{\Omega\left(x_{1}^{\prime}, x_{2}^{\prime}\right)}{\left|x_{1}\right|^{\beta_{1}}\left|x_{2}\right|^{\beta_{2}}} \chi_{\left\{\left|x_{1}\right| \leq t_{1},\left|x_{2}\right| \leq t_{2}\right\}}\left(x_{1}, x_{2}\right) .
$$

We can prove theorems similar to those in Section 4 with the obvious modifications: the $A_{p}$ weights of the Hardy-Littlewood maximal function are replaced by the $A_{p}^{*}$ weights of the strong maximal function and the maximal operator $M_{\Omega}$ of (10) is adapted to the product structure. In particular, when $\Omega$ is bounded the resulting square function is bounded on $L^{p}(w)$ for all $w \in A_{p}^{*}$. The proof of the decay estimates required for $\widehat{N}_{t_{1}, t_{2}}$ is similar to that in the one-parameter case; a proof for the singular integral case is in [4].

6.2. Non-isotropic dilations. The usual theory of Calderón-Zygmund operators has been developed in a more general context than Euclidean dilations on $\mathbb{R}^{n}$. For instance, we can consider a more general group of dilations and associate a pseudonorm to it as in [17]. Then the classical theory is extended in the sense of spaces of homogeneous type ([2]). All the ingredients we used in the proofs of our theorems have their counterpart in this context and the same type of general results can be obtained. We leave the details to the reader.

6.3. Other square functions. As mentioned in the introduction and can be seen in [15], several types of square functions have been studied. It is possible to define extensions with rough kernels for those related with the so-called area integral and $g_{\lambda}^{*}$ function.

Let again $\Omega$ be in $L^{q}\left(S^{n-1}\right)$ with mean value zero, and $b$ a bounded radial function. Define

$$
\mu_{\Omega, S}(f)(x)=\left(\int_{\Gamma(x)}\left|F_{t}(y)\right|^{2} \frac{d t}{t^{n+1}}\right)^{1 / 2}
$$

where $\Gamma(x)=\left\{(y, t) \in \mathbb{R}^{n} \times(0, \infty):|x-y|<t\right\}$, and for $\lambda>1$,

$$
\mu_{\Omega, \lambda}^{*} f(x)=\left(\int_{\mathbb{R}^{n}} \int_{0}^{\infty}\left(\frac{t}{t+|x-y|}\right)^{n \lambda}\left|F_{t}(y)\right|^{2} \frac{d y d t}{t^{n+1}}\right)^{1 / 2} .
$$

In both cases for each $t>0$ and some $\beta<n$,

$$
F_{t}(y)=\frac{1}{t^{n-\beta}} \int_{|y| \leq t} \frac{b(|y|) \Omega\left(y^{\prime}\right)}{|y|^{\beta}} f(x-y) d y .
$$


TheOREM 6. Let $p \geq 2$ and $w \in A W_{p}\left(M_{\Omega}\right) \cap A_{p / 2}$. Then both operators, $\mu_{\Omega, S}$ and $\mu_{\Omega, \lambda}^{*}$, are bounded on $L^{p}(w)$.

The proof of the theorem is based on the integral inequality

$$
\int_{\mathbb{R}^{n}}\left|\mu_{\Omega, \lambda}^{*}(f)(x)\right|^{2} w(x) d x \leq C_{\lambda} \int_{\mathbb{R}^{n}}|g(f)(x)|^{2} M w(x) d x
$$

proved in [19] for the smooth case and in [3] for the rough case and the pointwise inequality $\mu_{\Omega, S}(f)(x) \leq C_{\lambda} \mu_{\Omega, \lambda}^{*}(f)(x)$, which is easy to check. For the $L^{2}(w)$ result we assume first that $w \in A_{1}$ and then apply Theorem 1 . Due to this restriction extrapolation is only possible for $p>2$.

In particular, $w$ is in $A W_{p}\left(M_{\Omega}\right) \cap A_{p / 2}$ if either one of the following holds: (a) $\max \left(q^{\prime}, 2\right)=\alpha<p<\infty$ and $w \in A_{p / \alpha}$; (b) $2<p<q$ and $w^{1-(p / 2)^{\prime}} \in A_{p^{\prime} / q^{\prime}}$; (c) $2 \leq p<\infty$ and $w^{q^{\prime}} \in A_{p / 2}$. Those are the weights given in [3]. But our method produces more weights using $A W_{p}\left(M_{\Omega}\right)$, for instance, the weights $w(x)=|x|^{\alpha}$ satisfy the theorem when

$$
\max \left(-n,-1-(n-1) \frac{p}{q^{\prime}}\right)<\alpha<\min \left(n\left(\frac{p}{2}-1\right), p-1+(n-1) \frac{p}{q^{\prime}}\right),
$$

which gives (even for $p=2$ ) a larger range than the one deduced from the results in [3].

\section{References}

[1] A. Benedek, A. Calderón, and R. Panzone, Convolution operators on Banach space valued functions, Proc. Nat. Acad. Sci. U.S.A. 48 (1962), 356-365.

[2] R. R. Coifman and G. Weiss, Analyse harmonique non-commutative sur certains espaces homogènes, Lecture Notes in Math. 242, Springer, Berlin, 1971.

[3] Y. Ding, D. Fan and Y. Pan, Weighted boundedness for a class of rough Marcinkiewicz integrals, Indiana Univ. Math. J. 48 (1999), 1037-1055.

[4] J. Duoandikoetxea, Multiple singular integrals and maximal functions along hypersurfaces, Ann. Inst. Fourier 36 (1986), 185-206.

[5] - Weighted norm inequalities for homogeneous singular integrals, Trans. Amer. Math. Soc. 336 (1993), 869-880.

[6] —, Almost orthogonality and weighted inequalities, in: Contemp. Math. 189, Amer. Math. Soc., 1995, 213-226.

[7] J. Duoandikoetxea and J. L. Rubio de Francia, Maximal and singular integral operators via Fourier transform estimates, Invent. Math. 84 (1986), 541-561.

[8] J. Duoandikoetxea and L. Vega, Spherical means and weighted inequalities, J. London Math. Soc. 53 (1996), 343-353.

[9] J. García-Cuerva and J. L. Rubio de Francia, Weighted Norm Inequalities and Related Topics, North-Holland Math. Stud. 116, North-Holland, Amsterdam, 1985.

[10] B. Jawerth, Weighted norm inequalities: linearization, localization and factorization, Amer. J. Math. 108 (1986), 361-414.

[11] D. Kurtz, Littlewood-Paley and multiplier theorems on weighted $L^{p}$ spaces, Trans. Amer. Math. Soc. 259 (1980), 235-254. 
[12] J. L. Rubio de Francia, Factorization theory and $A_{p}$ weights, Amer. J. Math. 106 (1984), 533-547.

[13] S. Sato, Remarks on square functions in the Littlewood-Paley theory, Bull. Austral. Math. Soc. 58 (1998), 199-211.

[14] E. M. Stein, On the functions of Littlewood-Paley, Lusin and Marcinkiewicz, Trans. Amer. Math. Soc. 88 (1958), 430-466.

[15] —, The development of square functions in the work of Zygmund, Bull. Amer. Math. Soc. 7 (1982), 359-376.

[16] -, Harmonic Analysis: Real-Variable Methods, Orthogonality, and Oscillatory Integrals, Princeton Univ. Press, Princeton, 1993.

[17] E. M. Stein and S. Wainger, Problems in harmonic analysis related to curvature, Bull. Amer. Math. Soc. 84 (1978), 1239-1295.

[18] E. M. Stein and G. Weiss, Interpolation of operators with change of measures, Trans. Amer. Math. Soc. 87 (1958), 159-172.

[19] A. Torchinsky and S. Wang, A note on the Marcinkiewicz integral, Colloq. Math. 60-61 (1990), 235-243.

[20] D. K. Watson, Vector-valued inequalities, factorization and extrapolation for a family of rough operators, J. Funct. Anal. 121 (1994), 389-415.

Departamento de Matemáticas

Universidad del País Vasco

Euskal Herriko Unibertsitatea

Apartado 644

48080 Bilbao, Spain

E-mail: mtpduzuj@lg.ehu.es
Departamento de Matemática Aplicada E. T. S. de Arquitectura Universidad del País Vasco Euskal Herriko Unibertsitatea Apartado 799 20080 Donostia, Spain E-mail: mtpsehee@lg.ehu.es

Received July 27, 2000

Revised version February 12, 2001 